\title{
Collective cell guidance by cooperative intercellular forces
}

\author{
Dhananjay T. Tambe ${ }^{1 \dagger}$, C. Corey Hardin ${ }^{2 \dagger}$, Thomas E. Angelini ${ }^{3}$, Kavitha Rajendran ${ }^{1}$, \\ Chan Young Park' ${ }^{1}$, Xavier Serra-Picamal ${ }^{4}$, Enhua H. Zhou' ${ }^{1}$, Muhammad H. Zaman ${ }^{5}$, \\ James P. Butler', David A. Weitz ${ }^{3}$, Jeffrey J. Fredberg ${ }^{1 \star}$ and Xavier Trepat ${ }^{4 \star}$
}

Cells comprising a tissue migrate as part of a collective. How collective processes are coordinated over large multi-cellular assemblies has remained unclear, however, because mechanical stresses exerted at cell-cell junctions have not been accessible experimentally. We report here maps of these stresses within and between cells comprising a monolayer. Within the cell sheet there arise unanticipated fluctuations of mechanical stress that are severe, emerge spontaneously, and ripple across the monolayer. Within that stress landscape, local cellular migrations follow local orientations of maximal principal stress. Migrations of both endothelial and epithelial monolayers conform to this behaviour, as do breast cancer cell lines before but not after the epithelial-mesenchymal transition. Collective migration in these diverse systems is seen to be governed by a simple but unifying physiological principle: neighbouring cells join forces to transmit appreciable normal stress across the cell-cell junction, but migrate along orientations of minimal intercellular shear stress.

A variety of fundamental processes in development, health, and disease depend on the coordinated motion of cell groups $^{1-10}$. To describe coordinated cellular motions in these processes, high-throughput genomic approaches have identified molecular players and mapped their interaction into comprehensive signalling networks ${ }^{11,12}$. But even with detailed signalling and structural information in hand, the role of intercellular adhesion in collective migration is disputed ${ }^{13,14}$, and our understanding of collective cellular migration lacks predictive power and remains largely descriptive. Central to these limitations is the absence of a physical picture that links cell motion to mechanical stresses exerted within the cell body and at cell-cell boundaries, for these stresses have never before been measured. Here we report high-resolution maps of these stress components everywhere within an advancing monolayer sheet, which serves as a simple experimental model system. These stress maps reveal that the local cellular trajectory follows local stress fields that are severely heterogeneous and dramatically cooperative over distances spanning many cell bodies. Together, these findings reveal an unanticipated but unifying physiological principle, namely, that each cell tends to migrate and remodel so as to maintain minimal local intercellular shear stress. Detailed knowledge of the biology of the cell-cell junction, the cryptic lamellipodium (Supplementary Information S7), or any specific molecular event could never predict such a unifying principle because it is an emergent property of a multicellular collective system. By analogy to the well-known guidance mechanisms of chemotaxis, durotaxis and haptotaxis, we call this distinct but innately collective mechanism plithotaxis, from the Greek 'plithos' denoting crowd, swarm or throng.

To measure the local state of stress within a monolayer (Fig. 1), we developed monolayer stress microscopy, MSM (Supplementary
Information S1). On an inverted optical microscope, we record cell-generated displacements of fluorescent markers embedded near the surface of a collagen-coated polyacrylamide gel substrate on which the cells are adherent. We use a novel approach for stage drift compensation (Supplementary Information S1), and then use resulting dedrifted gel deformations to compute a map of the traction forces, $T$, exerted by the monolayer on the gel ${ }^{15}$. Finally, from these traction forces measured directly at the interface between the cell and its substrate (Supplementary Fig. S3), a straightforward and rigorous two-dimensional balance of forces as demanded by Newton's laws is then used to obtain the distribution of the mechanical line forces everywhere within the cell sheet (Fig. 1a); for convenience, these measured line forces (in units of force per unit length) are converted to stresses (force per unit area) using the average monolayer height, $h$ (Fig. 1; Supplementary Fig. S4). Gradients of these line forces and stresses within the cell sheet are attributable to the pile-up of traction forces applied on the underside of the cells. At each point within the sheet the local coordinate system (Fig. 1c) can be rotated in the cell plane to find those special orientations along which the local normal stress is maximal and minimal, respectively, thus defining the two principal stress components $\left(\sigma_{\max }\right.$ and $\left.\sigma_{\min }\right)$ and the two corresponding, mutually perpendicular, principal orientations (Fig. 1d; Supplementary Information S1). As such, the associated MSM result displays at high resolution, and maps separately, each individual component of the in-plane stress tensor.

We consider first the average local normal stress, simply defined as $\bar{\sigma}=\left(\sigma_{\max }+\sigma_{\min }\right) / 2$, and its spatial heterogeneity. A traditional image of an advancing monolayer of rat pulmonary microvascular endothelial (RPME) cells is unremarkable (Fig. 2a). The underlying distribution of local normal stress, by contrast, is

\footnotetext{
${ }^{1}$ Program in Molecular and Integrative Physiological Sciences, School of Public Health, Harvard University, Boston, Massachusetts 02115, USA, ${ }^{2}$ Division of Pulmonary and Critical Care Medicine, Massachusetts General Hospital, Boston, Massachusetts 02114, USA, ${ }^{3}$ School of Engineering and Applied Sciences, Harvard University, Cambridge, Massachusetts 02138, USA, ${ }^{4}$ Institute for Bioengineering of Catalonia, Universitat de Barcelona, Ciber Enfermedades Respiratorias, and Institució Catalana de Recerca i Estudis Avançats, 08036, Spain, ${ }^{5}$ Department of Biomedical Engineering, Boston University, Boston, Massachusetts 02215, USA. †These authors contributed equally to this work. *e-mail: jfredber@hsph.harvard.edu; xtrepat@ub.edu.
} 


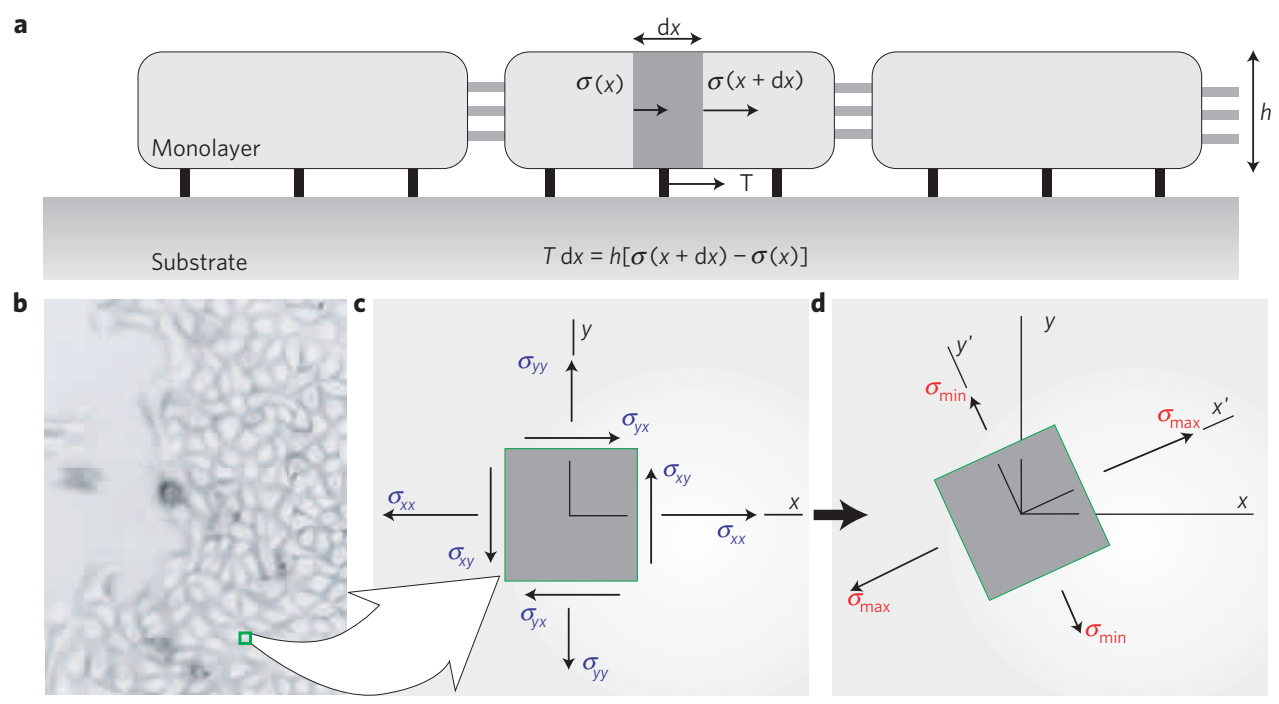

Figure 1 | Monolayer stress microscopy. a, Simplified representation of the physical relationship between cell-substrate tractions, $T$, which have been reported previously ${ }^{15}$, and intercellular stresses, $\sigma$, which are reported for the first time here. Intercellular stresses arise from the accumulation of unbalanced cell-substrate tractions. At any point within the monolayer $(\mathbf{b})$, the intercellular stresses, defined in laboratory frame $(x, y),(\mathbf{c})$, have shear $\left(\sigma_{x y}\right.$, and $\left.\sigma_{y x}\right)$ and normal $\left(\sigma_{x x}\right.$, and $\left.\sigma_{y y}\right)$ components. This frame can be rotated locally to obtain the principal frame $\left(x^{\prime}, y^{\prime}\right)$, (d), where shear stresses vanish and the resulting normal stresses are called principal stresses $\left(\sigma_{\max }\right.$ and $\left.\sigma_{\min }\right)$. The corresponding axes are called maximum, aligned with $x^{\prime}$, and minimum, aligned with $y^{\prime}$, principal orientations.

severely heterogeneous; normal stresses are mostly positive (tensile) with values exceeding $300 \mathrm{~Pa}$ in regions spanning tens of cells. These regions of predominantly tensile stresses alternate with regions of weakly negative (compressive) stresses (Fig. 2c). These fluctuations occur steadily over distances spanning multiple cell widths and define a stress landscape that is rugged (Fig. 2c,i), by which we mean that the spatial fluctuations over these relatively short distances are comparable in magnitude to the spatial mean values. We consider next the distribution of the intercellular shear stress (Supplementary Fig. S1), which is not to be confused with any additional shear stress that might be imposed by flow over the monolayer surface ${ }^{16}$, which in this case is everywhere zero. As in the case of the normal stress, the shear stress at a point within a material varies with orientation and attains its maximal value, $\mu=\left(\sigma_{\max }-\sigma_{\min }\right) / 2$, at $45^{\circ}$ from the principal orientations (Fig. 1d). The local maximal shear stress was systematically smaller than the local normal stress, but was also characterized by a rugged landscape (Fig. 2e). As the monolayer advances, these respective stress landscapes evolve continuously in time (Supplementary Movie SM1). Finally, the dependence of local stresses on orientation signifies stress anisotropy. To visualize this anisotropy, we plotted ellipses for which the major axis corresponds to the local $\sigma_{\max }$ and the minor axis corresponds to the local $\sigma_{\min }$, each aligned with the corresponding principal orientations. Where $\sigma_{\max }=\sigma_{\min }$ the stress field is isotropic, the ellipse becomes a circle, $\mu$ is zero, and there exists no preferred stress orientation. But where $\sigma_{\max } \gg \sigma_{\min }$ the local stress field is highly anisotropic, the ellipse becomes spindle-like, $\mu$ is nonzero, and there exists a strongly preferred and well-defined stress orientation. From region-to-region, we found that ellipse size, ellipse shape, and ellipse orientation varied extensively, but with strong local correlations (Fig. 2g).

As cells extend cryptic lamellipodia ${ }^{17}$ (Supplementary Fig. S7) and advance within the monolayer, stresses at every point and at every instant of time must be in mechanical balance. Nonetheless, no mechanistic framework or physical picture yet exists that might link these stresses to cellular orientation, remodelling, or migration. Here we ask, to what extent are these intercellular stresses meaningful biologically and useful predictively? The answer to this question is suggested by two pieces of experimental evidence. First, because phase-contrast images and stress maps are mutually independent measurements, the coincidence between the orientation of the cell body versus the orientation of the maximal principal stress is striking (Fig. $2 \mathrm{~g}$ and Supplementary Fig. S5). Further, because the maximal principal orientation corresponds to the local axis of highest normal stresses and zero shear stress, this result suggests that the cell-cell junction, as well as the cell body, supports high normal stresses, which are overwhelmingly tensile, but only minimal shear stresses. One would predict, therefore, that major organized actin structures that span the cell, as would be imaged at low resolution, might align with maximal principal orientations, and for the spindlelike RPME cells this is in fact seen to be the case (Fig. $2 \mathrm{~g}$ and Supplementary Fig. S6). Second, cells not only align with the maximal principal orientation, but also migrate along that orientation (Fig. 2g, red arrows; Supplementary Movie SM2). Appreciable portions of the stress field are approximately isotropic, however, and therefore the local orientation of cell motion would not be expected to correlate with a stress field possessing no preferred orientation.

As such, these observations lead naturally to the following prediction: regions of higher stress anisotropy will exhibit stronger alignment between the direction of local maximal principal stress and that of local cellular migration velocity. To test this prediction, we reasoned as follows. As the maximum local shear stress is given by $\mu=\left(\sigma_{\max }-\sigma_{\min }\right) / 2$, we took $\mu$ as a direct and quantitative index of stress anisotropy. We then rank-ordered this stress anisotropy by quintiles. For each point within the cellular monolayer falling within any given quintile, we measured the alignment angle $\phi$ between the orientation of the local maximal principal stress and the orientation of the local cellular migration velocity vector (Fig. 2 j, inset). The greater was the local shear stress, the narrower was the distribution of $\phi$ (Fig. $2 \mathrm{j}-1$ ). We then constructed the cumulative probability distribution function, $\bar{P}(\phi)$, reasoning that if there were perfect alignment between the orientation of local cellular migration velocity and that of local maximal principal stress, then all angles $\phi$ would be $0^{\circ}$ and the cumulative probability distribution would be a step function from 

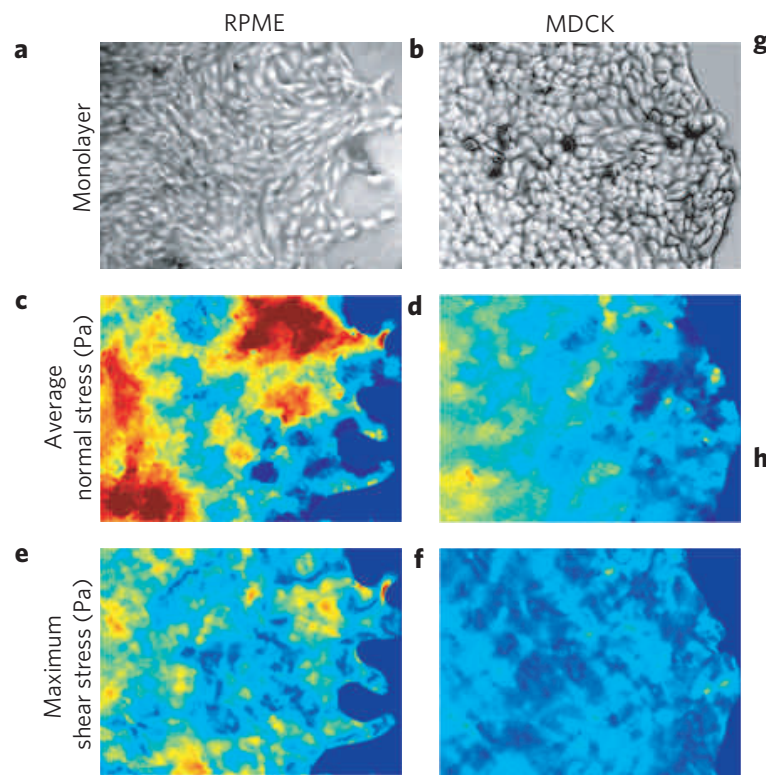

$0 \quad 100 \quad 200300$

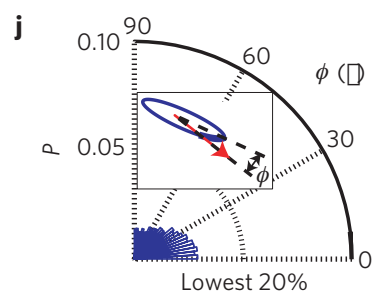

Shear stress quintiles (RPME)

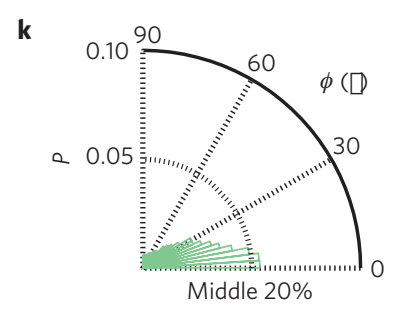

Shear stress quintiles (RPME)

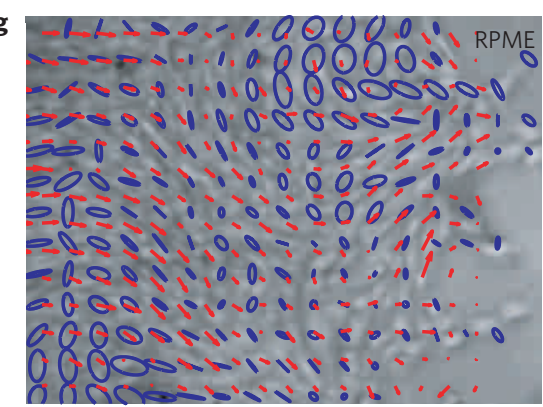

\section{h}

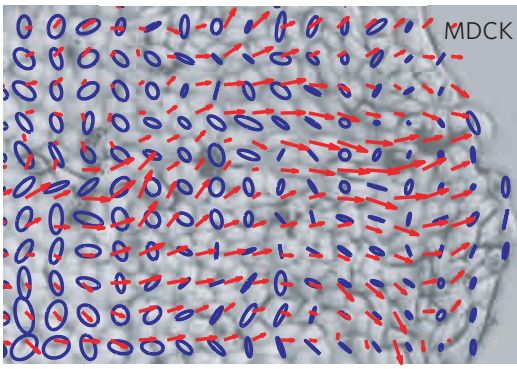

Stress ellipses and velocity vectors

\section{I}

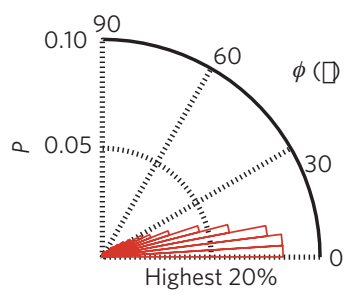

Shear stress quintiles (RPME)

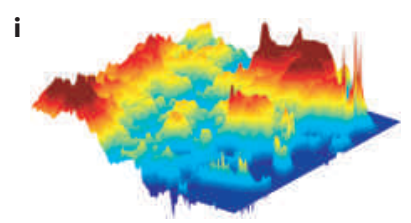

m

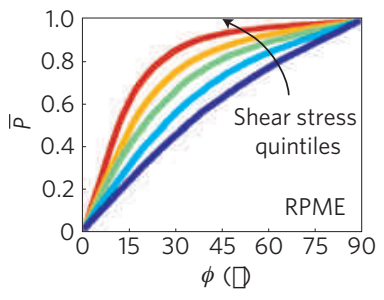

n

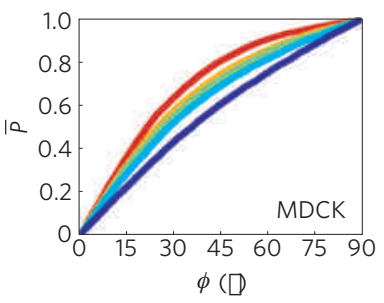

Figure 2 | Intercellular stress maps and mechanical guidance of collectively migrating monolayers. Transmitted light image of the RPME cell monolayer (a) and the MDCK cell monolayer (b). Corresponding to these images are the maps of average normal stress (c,d), maximum shear stress (e,f) and principal stress ellipses (blue) and cell velocity vectors (red) ( $\mathbf{g}, \mathbf{h}$ ). Note that for the MDCK cell monolayer, the average tensile stress (d) increased systematically with increasing distance from the advancing front, thus contributing to the state of global tug-of-war ${ }^{15}$. The map of average normal stress for the RPME cell monolayer is predominately tensile, but forms a rugged stress landscape (i). The alignment angle, $\phi$, between the major axis of the principal stress ellipse and the direction of the cellular motion ( $\mathbf{j}$, inset) shows that the greater the local maximum shear stress the narrower is the distribution of $\phi(\mathbf{j}-\mathbf{I})$. The cumulative probability distribution $\bar{P}(\phi)$ varied strongly and systematically with stress anisotropy (m); curves, from blue to red,

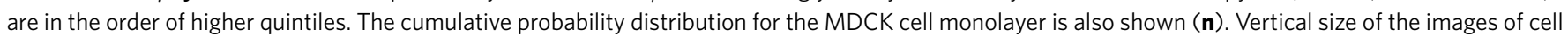
monolayers: RPME-545 $\mu \mathrm{m}$, MDCK-410 $\mu \mathrm{m}$. Each curve in $\mathbf{m}$ and $\mathbf{n}$, and distributions in $\mathbf{j}, \mathbf{k}$ and $\mathbf{I}$ have more than 8,000 observations.

probability 0 to probability 1 occurring at $0^{\circ}$ (Fig. $\left.2 \mathrm{~m}\right)$. If there were no alignment, however, then all angles between $0^{\circ}$ and $90^{\circ}$ would be equally likely, and the cumulative probability function would be a straight line from probability 0 at $0^{\circ}$ to probability 1 at $90^{\circ}$. In the regions with lowest stress anisotropy (blue), the angular distribution was broad but not uniform. In regions with highest stress anisotropy (red), the angular distribution was quite narrow; the orientation of cellular velocity and the orientation of maximal principal stress were coupled strongly, but were unrelated to the magnitude of local average stress (Supplementary Fig. S8). The stronger was the stress anisotropy the greater was the overall degree of alignment.

To assess the generality of this finding, we then examined monolayers comprising Madin-Darby canine kidney (MDCK) cells (Fig. 2b), which are of particular interest because they are epithelial, not endothelial, and because they are rounded in the plane, not spindle-shaped as are RPME cells. Despite these differences in cell type and cell morphology, the stresses were dramatically heterogeneous (Fig. 2d,f) and the local orientation of cellular migration was also found to follow the local orientation of maximal principal stress (Fig. 2h,n). Remarkably, local cell motions tended to follow local principal stress orientations even when local cell geometry displayed no preferred orientation. To assess further the generality of this finding, we next examined the behaviour of monolayers of well-established breast-cancer model systems: MCF10A cells (control or vector) (Fig. 3a), MCF10A cells overexpressing ErbB2/HER-2/neu (Fig. 3b), and MCF10A cells overexpressing 14-3-3 $\zeta$ (Fig. 3c). We chose these cell lines because each exhibits pronounced morphological differences as well as diverse levels of transforming potential, expression of cell-cell junction proteins, and cell proliferation ${ }^{18,19}$. Much as in the case of endothelial cells and control epithelial cells, ErbB2 cells moved in alignment with the direction of maximum principal stress (Fig. 3m). By contrast, 14-3-3 $\zeta$ cells, which have decreased expression of cell-cell junctional markers ${ }^{18,19}$, were seen to move nearly independently of the orientation of the maximum principal stress (Fig. 3m). To assess further the importance of cell-cell adhesion, we weakened the cell-cell contacts of MCF10A vector cells by calcium chelation (Fig. $4 \mathrm{~g}$,i). As expected, the alignment between the orientations of local stress and the orientations of local cellular motions was lessened (Fig. 4s, magenta), but was restored on returning to the normal growth medium (Fig. 4i,s, blue). However, this reversibility was blocked in the presence of E-cadherin antibodies (Fig. 4r,s, red). Together, these observations 

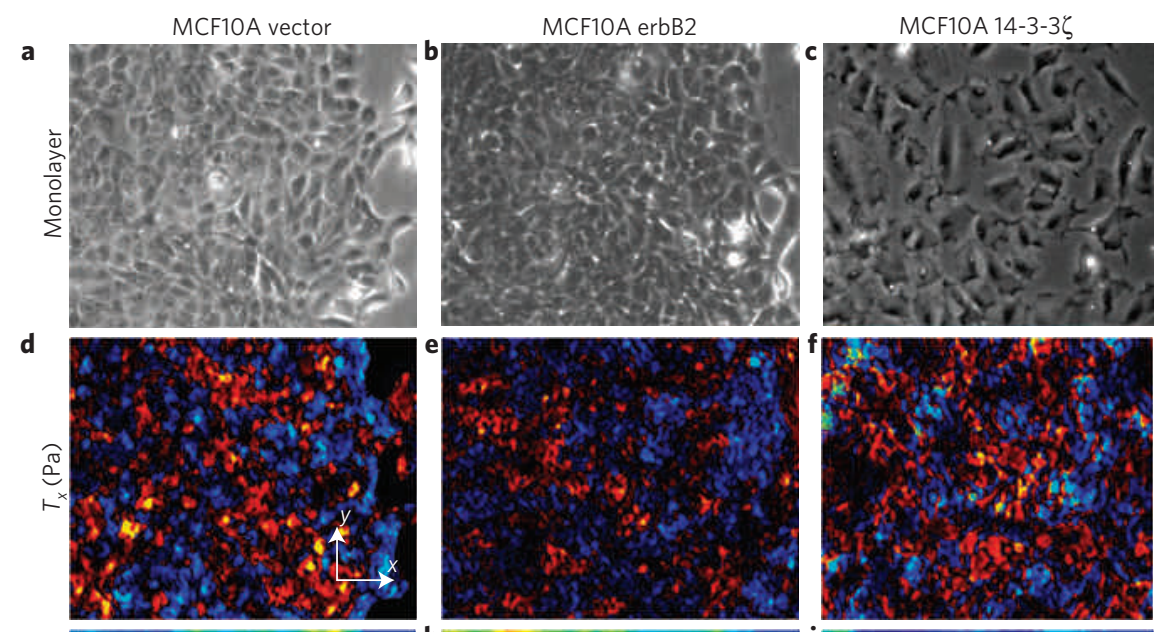

100
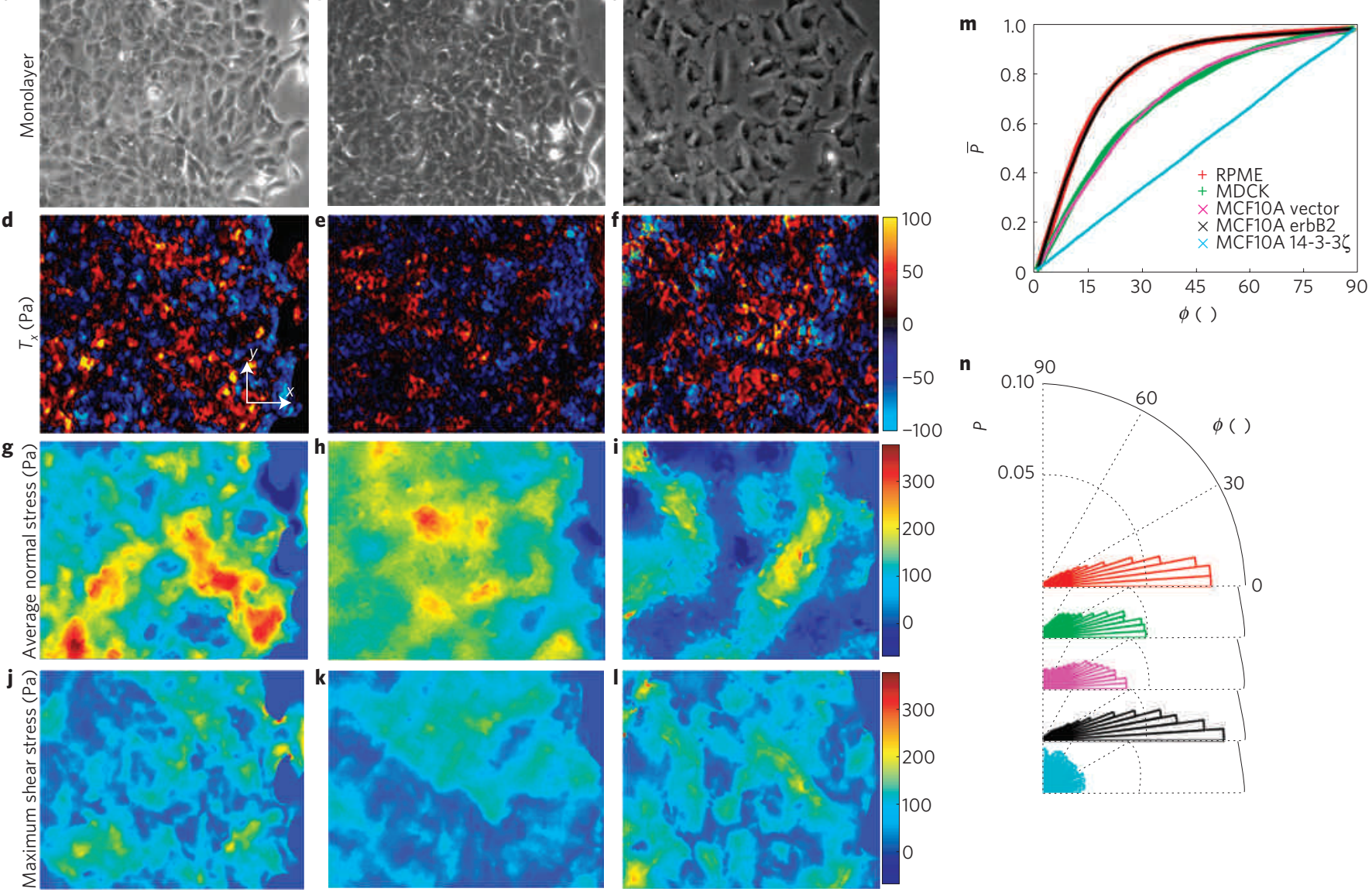

Figure 3 | Stress maps and migration in monolayers of breast-cancer model systems. Phase contrast image of nontransformed human mammary

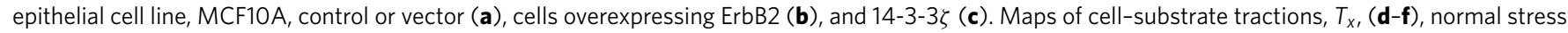
$(\mathbf{g}-\mathbf{i})$, and maximum shear stress $(\mathbf{j}-\mathbf{I})$ corresponding to each of these three mammary epithelial cell lines. $\mathbf{m}$, Cumulative probability distribution of $\phi$ for the regions corresponding to the highest quintile of the shear stress for five different cell sheets. $\mathbf{n}$, Distributions corresponding to the curves in $\mathbf{m}$. Vertical size of the images of monolayers: $410 \mu \mathrm{m}$. Each curve in $\mathbf{m}$ has more than 8,000 observations.

establish that transmission of mechanical stresses from cell-to-cell across many cells is necessary for plithotaxis, that is, for each individual cell to follow the local orientation of the maximal principal stress.

For collective migration to be coordinated across many cells, intercellular stresses might be expected to be cooperative over comparable distances; cooperativity of cell motions has been recently established ${ }^{20,21}$, but cooperativity of cellular stresses has not. To quantify the spatial extent of any such stress cooperativity, we first examined the spatial autocorrelation function of the average normal stress:

$$
C(R)=\frac{1}{N \operatorname{var}(\bar{\sigma})^{2}} \sum_{i, j=1\left|\mathbf{r}_{i}-\mathbf{r}_{j}\right|=R} \delta \bar{\sigma}_{i} \cdot \delta \bar{\sigma}_{j}
$$

where $\delta \bar{\sigma}_{i}$ is the local departure of the average normal stress at position $\mathbf{r}_{i}$ from its spatial mean $\left\langle\bar{\sigma}_{i}\right\rangle, \operatorname{var}(\bar{\sigma})$ is the variance of those departures, and the notation $\left|\mathbf{r}_{i}-\mathbf{r}_{j}\right|=R$ means equality within a uniform bin width of $5 \mu \mathrm{m}$. Confining the attention to regions many cell lengths from the leading edge of an MDCK monolayer (Fig. 5a), fluctuations in normal stress (Fig. 5c) were found to be correlated over a length scale of approximately $10-15$ cell diameters (Fig. 5e, blue). Cooperativity of normal stresses over 10-15 cell diameters might be attributable to alignment of principal stresses end-to-end, as in a tug-of war, or side-by-side, as police who lock arms during crowd control. To assess whether normal stresses are aligned according to either of these configurations, we decomposed the maximum principal stress into end-to-end and side-by-side contributions,

$$
\begin{aligned}
C_{\text {end }}(R) & =\frac{1}{N\|F\|^{2}} \sum_{i, j=1\left|\mathbf{r}_{i}-\mathbf{r}_{j}\right|=R}^{N} \mathbf{F}_{i} \cdot \mathbf{F}_{j} \cos ^{2} \theta_{i j} \\
C_{\text {side }}(R) & =\frac{1}{N\|F\|^{2}} \sum_{i, j=1\left|\mathbf{r}_{i}-\mathbf{r}_{\mathbf{j}}\right|=R}^{N} \mathbf{F}_{i} \cdot \mathbf{F}_{j} \sin ^{2} \theta_{i j}
\end{aligned}
$$

where $\|\cdots\|$ denotes $L^{2}$ norm, $F_{i}$ is the local maximal principal stress considered as a vector quantity (such that the angle between the maximal and minimal principal stress orientations is taken modulo $\pi$ ) and $\theta_{i j}$ is the angle between adjacent vector pairs. The two components were found to contribute almost equally to force cooperativity, thus indicating the coexistence of both end-to-end and side-by-side force correlations (Fig. 5f). Simply put, to move cooperatively, neighbouring cells join forces.

Cooperative motions emerge naturally in inert particulate systems that exhibit close-packing, structural disorder, and glassy dynamics, such as colloidal glasses ${ }^{22}$. A central feature that identifies these systems as being glassy is the slowing of internal structural rearrangement as system density is increased; with 

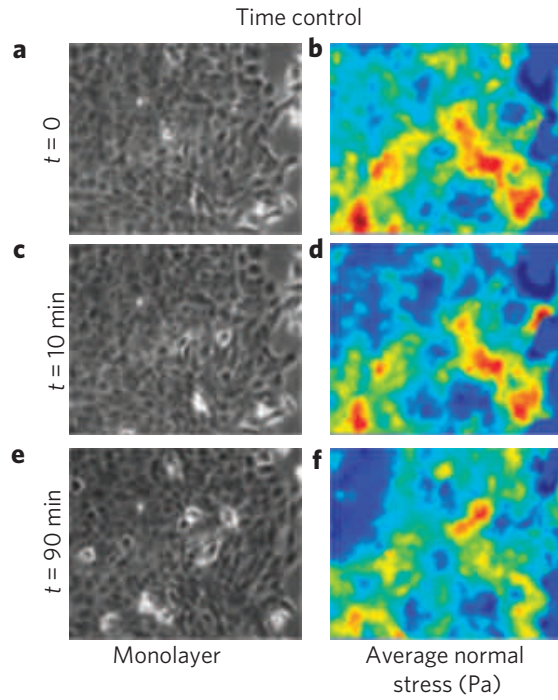

s

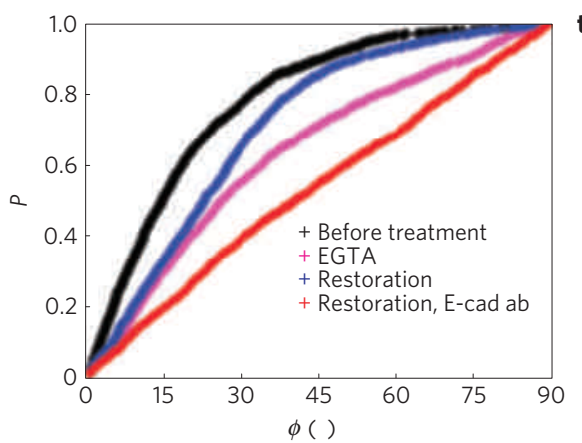

Chelation of calcium from medium reversibly attenuates intercellular stresses
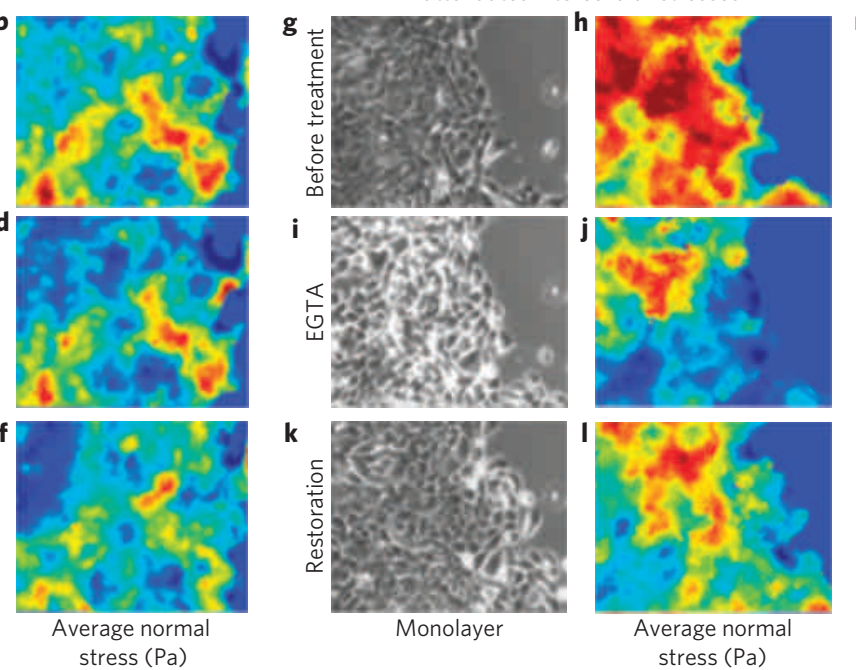

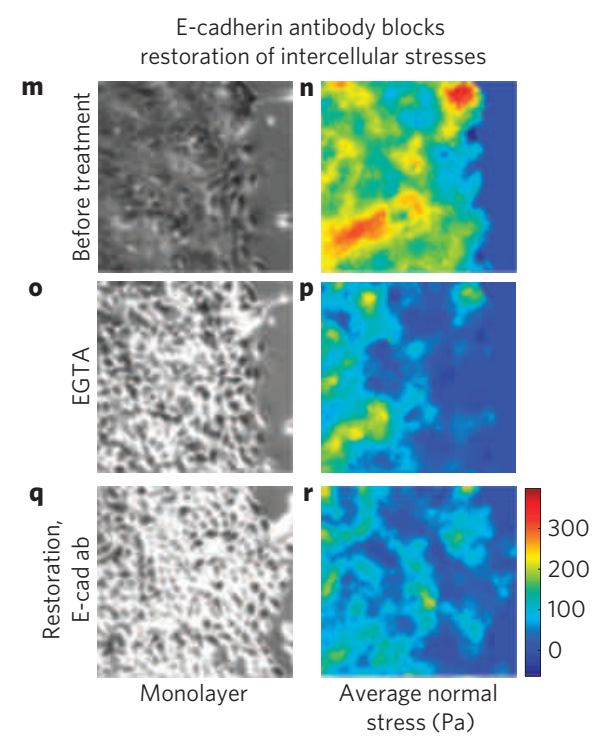

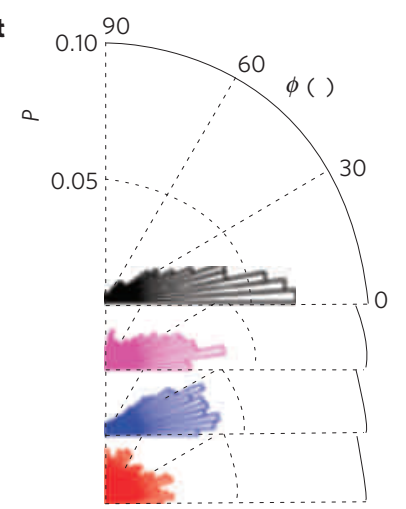

Figure 4 | Local cell guidance requires force transmission from cell-to-cell. Time-controls of intercellular stress maps of MCF10A-vector cell monolayers (a-f). The stress patterns do not change appreciably over a period of $80 \mathrm{~min}$. After $10 \mathrm{~min}$ in the presence of the calcium chelator ethylene glycol

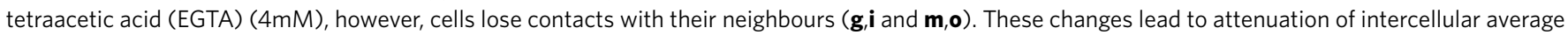

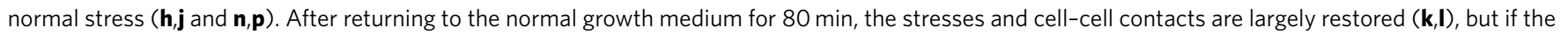
growth medium is supplemented with E-cadherin antibody $\left(7 \mu \mathrm{g} \mathrm{m}^{-1}\right)$ recovery of the stresses and cell-cell contacts is blocked (q,r). EGTA treatment widens the distribution of angle $(\phi)$ between the local cellular velocity and the local maximum principal orientation corresponding to highest of the maximum shear stress quintiles (s,t). The distribution of $\phi$ is narrowed if calcium is restored (s,t, blue), but widened further if the restoration medium is supplemented with E-cadherin antibody ( $\mathbf{s}$ and $\mathbf{t}$, red). Together, these data show that local cell guidance along the orientation of maximal principal stress (plithotaxis) requires force transmission across cell-cell junctions. These preferred orientations correspond to those engendering minimal intercellular shear stresses. Increased intensity at cell boundaries in phase contrast images (i,o, and $\mathbf{q}$ ) reveals disruption of cell-cell junctions. Vertical size of the images of monolayers: $410 \mu \mathrm{m}$. Each data set in $\mathbf{s}$ and $\mathbf{t}$ has more than 1,500 observations.

increasing system density, each particle becomes increasingly trapped by its neighbours so that, to rearrange at all, many neighbouring particles must rearrange cooperatively ${ }^{23}$. As such, the size of cooperative clusters increases as system density increases. Moreover, as the size of the cluster grows the number of possible structural rearrangements decreases and, as such, the time needed for cooperative rearrangements increases precipitously until, eventually, the system becomes virtually frozen, or stuck ${ }^{23}$. Cooperative cellular motions within the monolayer sheet exhibit these very signatures of glassy dynamics ${ }^{24,25}$, but to what extent might cellular stresses depict a complementary physical picture? To answer this question we analysed the motion of the MDCK monolayers as cellular density increased with the passage of time $^{15,20}$. Consistent with an expectation of glassy dynamics, the spatial decay in $C(r)$ was smaller when the density was greater (Fig. 5e, red curve with corresponding monolayer and force map Fig. 5b,d), indicating that force cooperativity extended to greater distances. As a direct measure of slowing of structural rearrangements we turned to metrics commonly used in soft condensed matter systems. We consider the average number of cells which change position between two points in time, which defines an overlap function $q_{\mathrm{s}}$ :

$$
q_{\mathrm{s}}=\frac{1}{N} \sum_{1}^{N} w\left(\left|\mathbf{r}_{i}(t)-\mathbf{r}_{i}(t=0)\right|\right)
$$

where the weight function $w$ is equal to one if the distance between cell positions at sequential times is less than half a cell diameter, and zero otherwise. The variance of $q_{\mathrm{s}}$ is then a measure of the rate of overall structural rearrangement ${ }^{26}$ and is related to the so-called four-point susceptibility $\chi^{\text {ss }}$. The peak in $\chi^{\text {ss }}$ occurs at the overall structural relaxation time, and the height of that peak is related to the size of rearranging regions ${ }^{27,28}$. If the system is glassy, the peak in $\chi^{\text {ss }}$ is expected to shift towards longer times as system density is increased, and a clear shift of the peak in the more dense system confirms this expectation (Fig. 5g). The peak height also increases in the more dense system, confirming the presence of growing velocity clusters. Moreover, 


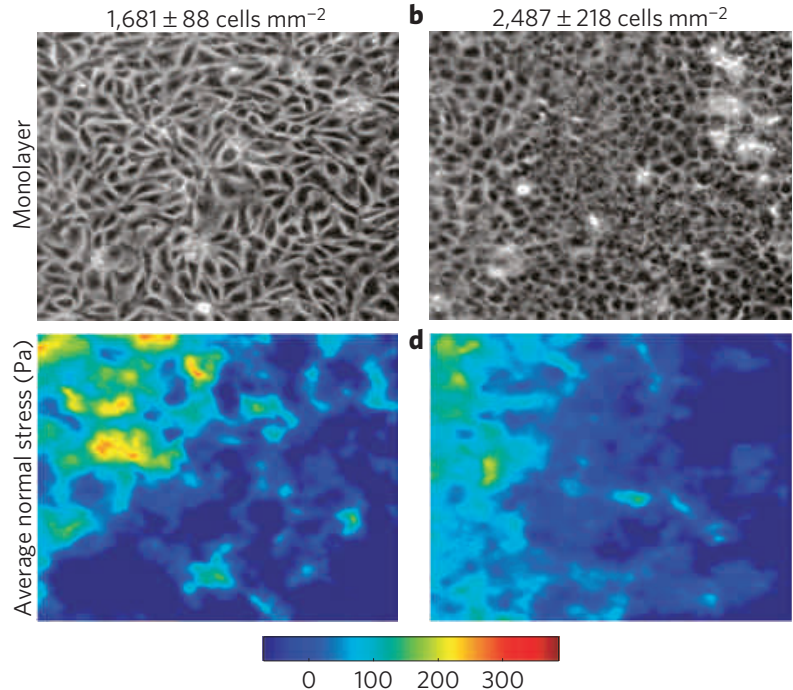

e
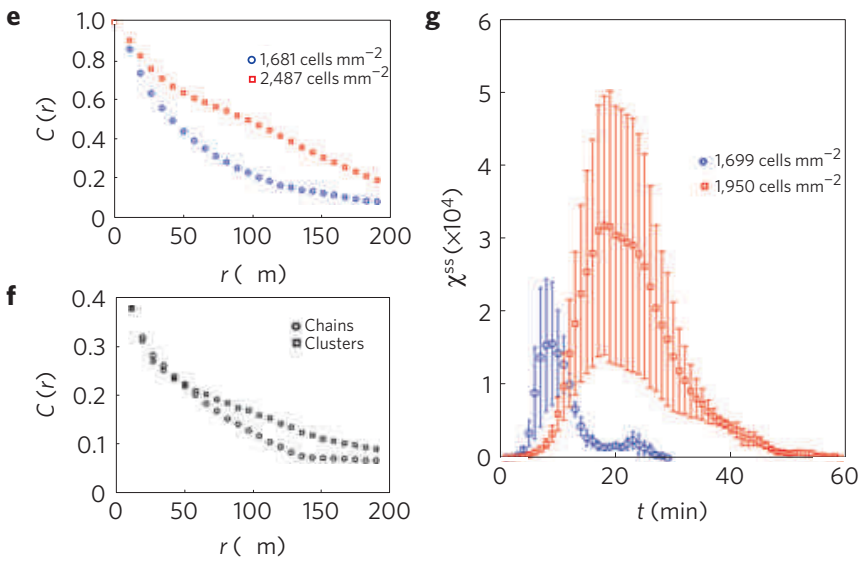

Figure $\mathbf{5}$ | Signatures of cooperativity and associated glassy dynamics.

Phase contrast images of a monolayer of MDCK cells well away from the leading edge at early (a, $t=196 \mathrm{~min}$, density $=1,681 \pm 88$ cells $\mathrm{mm}^{-2}$ ) and late $\left(\mathbf{b}, t=3,196 \mathrm{~min}\right.$, density $=2,487 \pm 218$ cells $\mathrm{mm}^{-2}$ ) times. Also shown are corresponding maps of average normal stress $(\mathbf{c}, \mathbf{d})$. Note that any contribution to the stress field with a wavelength longer than the size of the field of view is not included in the calculation. Thus a stress build up extending over the entire monolayer as previously reported ${ }^{15}$ is absent from this analysis. e, Time-averaged spatial autocorrelation function, $C(r)$, of average normal stress in low-density $\left(1,681\right.$ cells $\mathrm{mm}^{-2}$, blue $)$ and high-density $\left(2,487\right.$ cells $\mathrm{mm}^{-2}$, red) regions. $\mathbf{f}, C(r)$ of high-density maximal principal stress resolved into components representing force chains (circles) and force clusters (squares). $\mathbf{g}$, Variance, $\chi^{\text {ss }}$, of the self-overlap parameter, $q_{s}$, as a function of time, in early, low-density ( $t=1-270 \mathrm{~min}, 1,699 \pm 40$ cells $\mathrm{mm}^{-2}$, blue) and late, high-density ( $t=1,800-2,070 \mathrm{~min}, 1,950 \pm 156$ cells $\mathrm{mm}^{-2}$, red) intervals. Each curve represents an average over three successive $90 \mathrm{~min}$ windows of similar density. Error bars represent the standard deviation over the square root of the number of windows. Vertical size of the images of the monolayer: $480 \mu \mathrm{m}$.

these density-dependent shifts in the position and the peak height of $\chi^{\text {ss }}$, which are indicative of slowing of structural rearrangements, occur simultaneously with growth of force clusters, as indicated by the slowing decay in the force autocorrelation function with increasing density (Fig. 5e, red). Although a mechanistic link between inter-particle forces and spatially heterogeneous dynamics in glassy systems remains unclear ${ }^{29-31}$, the findings of Fig. 5 are consistent with an approach to a glass transition (Supplementary Information S9).
Recent advances have unravelled important features of stress transmission across specific molecular constituents of the focal adhesion and of the adherens junction, including vinculin, talin, and $\alpha$-catenin for example $e^{14,32-37}$, but the integrative context of these molecular events within integrated stress-bearing structures comprising highly redundant molecular pathways, or even across multi-cellular assemblies at larger scales of organization, have remained largely ambiguous. Logically, associated integrative principles have remained unstudied. Because distinct stress tensor components between contiguous cells in any complex living system have never before been measured, monolayer stress microscopy now sets the study of underlying molecular events within an integrative mechanical context that is conceptually comprehensive and experimentally rigorous. The finding that each cell comprising a monolayer tends to migrate and remodel so as to maintain minimal local intercellular shear stress complements other integrative physiological principles (Supplementary Information S10).

A central question in morphogenesis and disease is how differentiated structures emerge from homogeneous cell populations ${ }^{38}$. Differentiation and pattern formation in multi-cellular systems is currently explained by the existence of morphogen gradients and by local variations in the composition, topology, and stiffness of the extracellular matrix ${ }^{39}$. In addition, once transduced by the sensory machinery of the individual cell $^{40}$, the spontaneously emergent rugged stress landscape reported here would be expected to trigger non-uniform secretion of soluble or insoluble factors, thus altering the local cellular microenviroment, causing cytoskeletal reinforcement ${ }^{41}$ or cytoskeletal fluidization ${ }^{42,43}$, as well as activating in a highly non-uniform fashion stress-dependent genetic programs that give rise to differentiated tissues. These emergent stress heterogeneities are severe and persistent, but unanticipated. How they might become harnessed and regulated during morphogenesis or repair and, perhaps more importantly, how they might become unharnessed or dysregulated during disease or injury, we identify here as major open questions, but ones that are now accessible to direct experimental attack.

Received 27 November 2010; accepted 12 April 2011; published online 22 May 2011

\section{References}

1. Kumar, S. \& Weaver, V. M. Mechanics, malignancy, and metastasis: The force journey of a tumor cell. Cancer Metastasis Rev. 28, 113-127 (2009).

2. Butcher, D. T., Alliston, T. \& Weaver, V. M. A tense situation: Forcing tumour progression. Nat. Rev. Cancer 9, 108-122 (2009).

3. Discher, D. et al. Biomechanics: Cell research and applications for the next decade. Ann. Biomed. Eng. 37, 847-859 (2009).

4. Levental, K. R. et al. Matrix crosslinking forces tumor progression by enhancing integrin signaling. Cell 139, 891-906 (2009).

5. Paszek, M. J. \& Weaver, V. M. The tension mounts: Mechanics meets morphogenesis and malignancy. J. Mammary Gland Biol. Neoplasia 9, 325-342 (2004).

6. Bianco, A. et al. Two distinct modes of guidance signalling during collective migration of border cells. Nature 448, 362-365 (2007)

7. Friedl, P. \& Gilmour, D. Collective cell migration in morphogenesis, regeneration and cancer. Nat. Rev. Mol. Cell Biol. 10, 445-457 (2009).

8. Giampieri, S. et al. Localized and reversible TGF $\beta]$ signalling switches breast cancer cells from cohesive to single cell motility. Nature Cell Biol. 11, 1287-1296 (2009).

9. Montell, D. Morphogenetic cell movements: Diversity from modular mechanical properties. Science 322, 1502-1505 (2008).

10. Shaw, T. J. \& Martin, P. Wound repair at a glance. J. Cell Sci. 122, 3209-3213 (2009).

11. Simpson, K. J. et al. Identification of genes that regulate epithelial cell migration using an siRNA screening approach. Nature Cell Biol. 10, 1027-1038 (2008).

12. Vitorino, P. \& Meyer, T. Modular control of endothelial sheet migration. Genes Dev. 22, 3268-3281 (2008).

13. Bindschadler, M. \& McGrath, J. L. Sheet migration by wounded monolayers as an emergent property of single-cell dynamics. J. Cell Sci. 120, 876-884 (2007). 
14. Liu, Z. et al. Mechanical tugging force regulates the size of cell-cell junctions. Proc. Natl Acad. Sci. USA 107, 9944-9949 (2010).

15. Trepat, X. et al. Physical forces during collective cell migration. Nature Phys. 5, 426-430 (2009).

16. DePaola, N., Gimbrone, M. Jr, Davies, P. \& Dewey, C. Jr Vascular endothelium responds to fluid shear stress gradients. Arteriosclerosis Thrombosis 12, 1254-1257 (1992).

17. Farooqui, R. \& Fenteany, G. Multiple rows of cells behind an epithelial wound edge extend cryptic lamellipodia to collectively drive cell-sheet movement. J. Cell Sci. 118, 51-63 (2005).

18. Lu, J. et al. Breast cancer metastasis: Challenges and opportunities. Cancer Res. 69, 4951-4953 (2009).

19. Muthuswamy, S. K., Li, D., Lelievre, S., Bissell, M. J. \& Brugge, J. S. ErbB2, but not ErbB1, reinitiates proliferation and induces luminal repopulation in epithelial acini. Nature Cell Biol. 3, 785-792 (2001).

20. Angelini, T. E., Hannezo, E., Trepat, X., Fredberg, J. J. \& Weitz, D. A. Cell migration driven by cooperative substrate deformation patterns. Phys. Rev. Lett. 104, 168104 (2010).

21. Szabó, B. et al. Phase transition in the collective migration of tissue cells: Experiment and model. Phys. Rev. E 74, 061908 (2006).

22. Parisi, G. \& Zamponi, F. Mean-field theory of hard sphere glasses and jamming. Rev. Mod. Phys. 82, 789-845 (2010).

23. Weeks, E. R., Crocker, J. C., Levitt, A. C., Schofield, A. \& Weitz, D. A. Three-dimensional direct imaging of structural relaxation near the colloidal glass transition. Science 287, 627-631 (2000).

24. Angelini, T. E. et al. Glass-like dynamics of collective cell migration. Proc. Natl Acad. Sci. USA 108, 4714-4719 (2011).

25. Garrahan, J. P. Dynamic heterogeneity comes to life. Proc. Natl Acad. Sci. USA 108, 4701-4702 (2011).

26. Berthier, L. et al. Direct experimental evidence of a growing length scale accompanying the glass transition. Science 310, 1797-1800 (2005).

27. Keys, A., Abate, A., Glotzer, S. C. \& Durian, D. J. Measurement of growing dynamical length scales and prediction of the jamming transition in granular material. Nature Phys. 3, 260-264 (2007).

28. Toninelli, C., Wyart, M., Berthier, L., Biroli, G. \& Bouchaud, J-P. Dynamical susceptibility of glass formers: Contrasting the predictions of theoretical scenarios. Phys. Rev. E 71, 041505 (2005).

29. Hall, R. W. \& Wolynes, P. G. Intermolecular forces and the glass transition. J. Phys. Chem. B 112, 301-312 (2007).

30. Mueth, D. M., Jaeger, H. M. \& Nagel, S. R. Force distribution in a granular medium. Phys. Rev. E 57, 3164-3169 (1998).

31. Trappe, V., Prasad, V., Cipelletti, L., Segre, P. N. \& Weitz, D. A. Jamming phase diagram for attractive particles. Nature 411, 772-775 (2001).

32. del Rio, A. et al. Stretching single talin rod molecules activates vinculin binding. Science 323, 638-641 (2009).

33. Grashoff, C. et al. Measuring mechanical tension across vinculin reveals regulation of focal adhesion dynamics. Nature 466, 263-266 (2010).

34. Hu, K., Ji, L., Applegate, K. T., Danuser, G. \& Waterman-Storer, C. M. Differential transmission of actin motion within focal adhesions. Science 315, 111-115 (2007).
35. le Duc, Q. et al. Vinculin potentiates E-cadherin mechanosensing and is recruited to actin-anchored sites within adherens junctions in a myosin II-dependent manner. J. Cell Biol. 189, 1107-1115 (2010).

36. Rajfur, Z., Roy, P., Otey, C., Romer, L. \& Jacobson, K. Dissecting the link between stress fibres and focal adhesions by CALI with EGFP fusion proteins. Nature Cell Biol. 4, 286-293 (2002).

37. Yonemura, S., Wada, Y., Watanabe, T., Nagafuchi, A. \& Shibata, M. $\alpha$-Catenin as a tension transducer that induces adherens junction development. Nature Cell Biol. 12, 533-542 (2010).

38. Engler, A. J., Humbert, P. O., Wehrle-Haller, B. \& Weaver, V. M. Multiscale modeling of form and function. Science 324, 208-212 (2009).

39. Le Goff, L. \& Lecuit, T. Gradient scaling and growth. Science 331, 1141-1142 (2011)

40. Geiger, B., Spatz, J. P. \& Bershadsky, A. D. Environmental sensing through focal adhesions. Nature Rev. Mol. Cell Biol. 10, 21-33 (2009).

41. Roca-Cusachs, P., Gauthier, N. C., del Rio, A. \& Sheetz, M. P. Clustering of $\alpha 5 \beta 1$ integrins determines adhesion strength whereas $\alpha v \beta 3$ and talin enable mechanotransduction. Proc. Natl Acad. Sci. USA 106, 16245-16250 (2009).

42. Krishnan, R. et al. Reinforcement versus fluidization in cytoskeletal mechanoresponsiveness. PLoS ONE 4, e5486 (2009).

43. Trepat, X. et al. Universal physical responses to stretch in the living cell. Nature 447, 592-595 (2007).

\section{Acknowledgements}

For their critical comments, we thank R. Hubmayr (Mayo Clinic), R. Phillips (CalTech), D. Navajas (University of Barcelona), L. B. Freund (Brown University), D. Tschumperlin (Harvard University), C. Forbes Dewey, Jr (MIT) and V. B. Shenoy (Brown University). We acknowledge the support of the European Research Council (Starting Grant FP7/ERC-242993), the Spanish Ministry of Science and Innovation (BFU2009-07595) and the National Institutes of Health (R01HL102373, R01HL107561, R01CA132633). We thank D. Yu (MDACC) for the kind gift of MCF-10A cell lines.

\section{Author contributions}

D.T.T. developed algorithms and performed stress measurements. C.C.H. analysed data pertaining to force chains and glassy dynamics. D.T.T. and T.E.A. performed measurements of cell motions. K.R. and C.Y.P. assisted in protocol design and optimization. C.Y.P. performed staining of actin cytoskeleton. X.S-P. performed additional stress measurements on MDCK cells. M.H.Z. provided cancer cell lines and assisted with related data interpretation. D.T.T. and E.H.Z. made early conceptual contributions. J.P.B., D.A.W., J.J.F. and X.T. guided data interpretation and analysis. D.T.T., C.C.H., J.P.B., X.T. and J.J.F. wrote the manuscript.

\section{Additional information}

The authors declare no competing financial interests. Supplementary information accompanies this paper on www.nature.com/naturematerials. Reprints and permissions information is available online at http://www.nature.com/reprints. Correspondence and requests for materials should be addressed to J.J.F. or X.T. 